\title{
Chiari Network - Just an Innocent Bystander or Can Have Serious Impact on Clinical Outcomes
}

\author{
Anum Asif ${ }^{1, \text { *, Nauman Farooq }}{ }^{1}$, Tayebah Mumtaz ${ }^{2}$ \\ ${ }^{1}$ Department of Medicine, University of Pittsburgh Presbyterian Hospital, Pittsburgh, USA \\ ${ }^{2}$ Department of Medicine, Geisinger Medical Center, Danville, USA
}

Email address:

asifa@upmc.edu (A. Asif)

${ }^{*}$ Corresponding author

To cite this article:

Anum Asif, Nauman Farooq, Tayebah Mumtaz. Chiari Network - Just an Innocent Bystander or Can Have Serious Impact on Clinical Outcomes. American Journal of Internal Medicine. Vol. 7, No. 2, 2019, pp. 33-35. doi: 10.11648/j.ajim.20190702.12

Received: March 4, 2019; Accepted: March 14, 2019; Published: May 30, 2019

\begin{abstract}
Chiari network, a remnant of sinus venosus is often incidentally diagnosed on routine echocardiograms. Chiari network occurs due to incomplete resorption of right valve of sinus venosus. It is often noticed as fenestrated membranous structure or reticular network like structure in the valve of inferior vena cava and coronary sinus. The structure was first described by Von Rokitansky in 1875 and gained its name after pathologist Hans Chiari in 1897 when he described the structure in 11 cadavers. There are inconsistencies in the exact prevalence of this structure and has been reported in literature from 2-13.6\%. Most of this data is available from cadaveric or autopsied hearts. These structures are usually considered as benign congenital malformations but literature has reported various cases which have resulted in medical complications including endocarditis, thromboembolism or procedural difficulties. These structures can also mimic right atrial pathologies or thrombus creating a diagnostic challenge. It is important that clinicians are aware of these since misdiagnosis can have significant effect on clinical course and outcomes of the patients. The possibility of the presence of these congenital remnants should be kept in mind during clinical decision making. We present two clinical scenarios with incidental diagnosis of Chiari network and its effect on the clinical course of these patients.
\end{abstract}

Keywords: Chiari Network, Thrombus, Echocardiogram

\section{Introduction}

Chiari network, a remnant of sinus venosus, is considered clinically insignificant however it is often misdiagnosed as right atrial pathologies or thrombus. There have been various case reports in literature discussing its association with paradoxical embolism, infective endocarditis and arrythmias where as others are talking about its possible protective effect to prevent pulmonary embolism due to its fenestrated sieve-like structure at cavo-atrial junction. Sometimes it can possess diagnostic confusion since it mimics other right atrial pathologies. This can have serious impacts on clinical outcomes of the patients. We discuss two clinical scenarios with incidental findings of Chiari network and its implication.

\section{Case Presentation}

Case 1: An 81-year-old female with COPD and stage III A squamous cell lung cancer was admitted with worsening dyspnea and intubated for hypoxic hypercapnic respiratory failure. During her course of hospitalization, a transthoracic echocardiogram (TTE) was performed which showed a suspicious mass in the right atrium [Figure 1]. Given her increased thrombosis risk due to underlying malignancy, the diagnosis of right atrial clot was entertained; however, based on radiological suspicion by the cardiologist it was decided to further assess this mass by a trans-esophageal echocardiogram (TEE). TEE demonstrated a fenestrated, highly mobile, and echogenic structure suggesting the presence of Chiari network [Figure 2]. The accurate and timely evaluation spared the patient from unnecessary prolonged anticoagulation. 


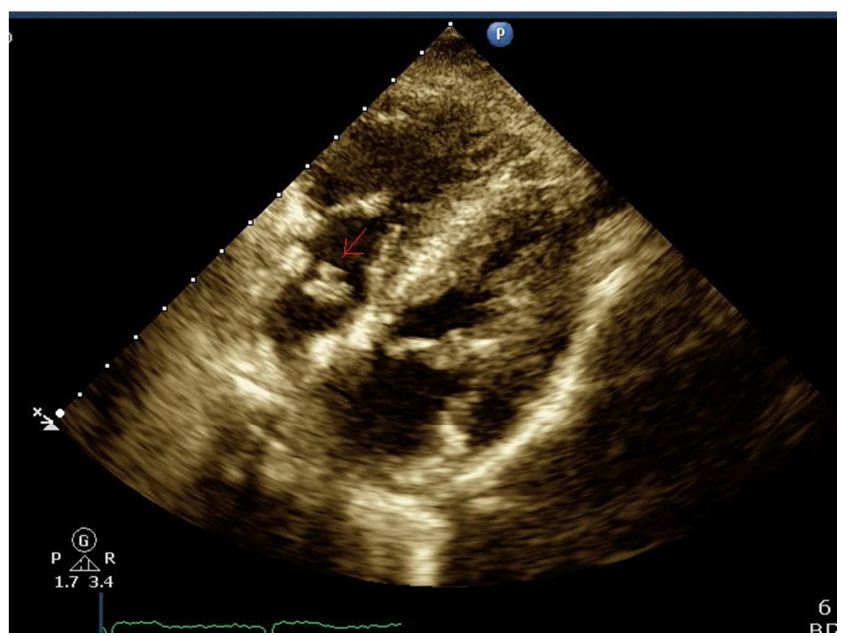

Figure 1. TTE showing a mass in right atrium.

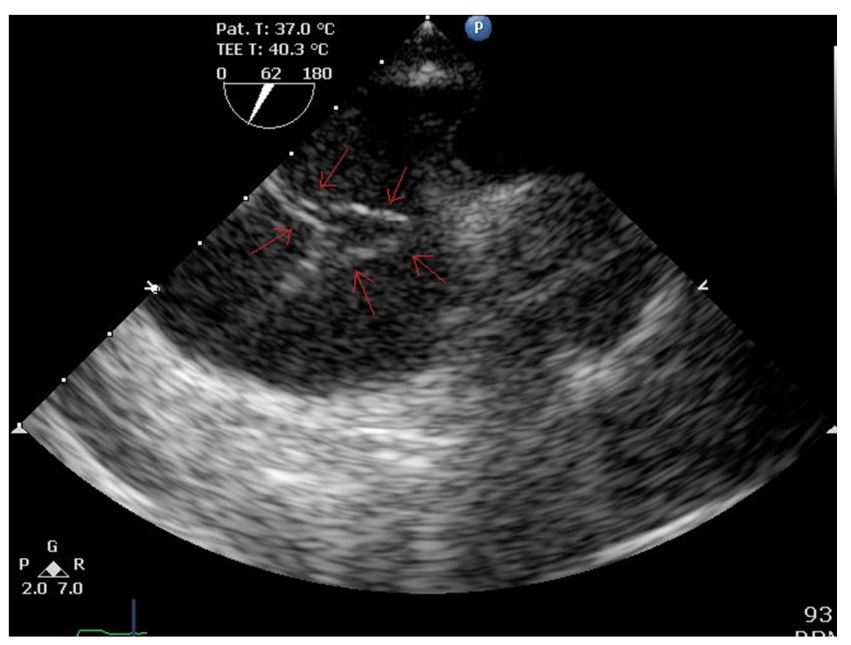

Figure 2. TEE showing a thread like structure in right atrium which was previously seen as a mass.

Case2: A 54-year-old female with prior history of chronic anticoagulation (AC) with warfarin for presumed right atrial (RA) thrombus on TTE and prior history of gastrointestinal bleed (GIB) complicated by hemorrhagic shock was admitted with non-ST segment elevation myocardial infarction (NSTEMI). She eventually had left heart catheterization and a drug eluting stent (DES) was placed in Left Circumflex artery. TTE done at this time again demonstrated persistent right atrial (RA) thrombus. The new diagnosis of NSTEMI with DES in the setting of prior hemorrhagic shock due to GIB brought clinical dilemma relating to anticoagulation status dual vs triple anticoagulation therapy. The fact that despite being on AC for many years, the presumed thrombus did not resolve at all led to further evaluation with a TEE which confirmed the presence of Chiari network mimicking a thrombus all those years. She was started on dual antiplatelet and warfarin was stopped.

\section{Discussion}

Chiari network is a congenital remnant of the right valve of the sinus venosus and septum spurium [1].
During the cardiac development, the two valves in the venous confluence of right atrium are secured by septum spurium. During development, these structures regress. Failure to do so results in a fenestrated membrane called Chiari network [2] There are inconsistencies in exact prevalence of this structure and has been reported in literature from $2-13.6 \%$. Most of this data is available from cadaveric or autopsied hearts [3]. It has been associated with underlying patent foramen ovale $(83 \%)$ and septal aneurysms (24\%) [4]

It was first discovered during autopsies as fenestrated membranous structure or reticular network in the valve of the inferior vena cava and coronary sinus. The structure was first described by Von Rokitansky in 1875 and gained its name after pathologist Hans Chiari in 1897 when he described the structure in 11 cadavers [5] It is mostly diagnosed incidentally during routine testing [6] and can mimic ruptured chords, thrombus, or intracardiac masses on imaging [7]. Chiari network does not have any clinical significance, but it has been reported in literature to be associated with infective endocarditis, thromboembolic phenomena [8] and procedural complications by catheter entrapment in these structures $[9,10]$. It has been reported to be involved in causing abnormal atrial depolarization and predisposes to arrhythmias. [11]. One of these fatal arrhythmias addressed in literature is atrial fibrillation which can lead to ischemic cerebrovascular accidents in patients [12]. Echocardiography is widely used for assessment of various cardiac structures and left ventricular function. These anomalous structures sometimes are routinely seen on these studies incidentally. Recently, 3D transthoracic echocardiogram has been found useful in making a definitive diagnosis of the Chiari network and differentiating it from other structures in the right atrium such as the Eustachian and Thebesian valves [13]. Cardiac magnetic resonance plays complimentary roles with echocardiography in differentiating Chiari network from right atrial pathologies. The cost and lack of facility at every hospital limit its routine use. [14]

Chiari network is an uncommon diagnosis, but it needs to be identified and recognized appropriately as the misdiagnosis can expose patients to the risk of unnecessary treatment leading to significant impact on the clinical course and outcomes. This is particularly important in our cases where the first patient could have diagnosis bias for thrombus in the setting of her underlying cancer history while our second patient was inappropriately treated with anticoagulation because of misdiagnosis and even had an adverse outcome of significant GI bleeding secondary to it. We suggest that a formal TEE should be encouraged in cases of clinical suspicion or where the size of mass/thrombus does not change despite anticoagulation. Although, TEE in every patient with atrial clot is not possible and might lead to unnecessary invasive imaging, interval improvement in thrombus burden should be evaluated with TTE in selected patient population with bleeding complications. Given the 
low prevalence of such anomaly, physicians should be encouraged to look for alternate diagnosis in patients such as described above. The cost of unnecessary interventions and their complications alone should be considered while making clinical decisions. It is pertinent to keep open mind and understanding of these anomalous structures and their relative clinical significance.

\section{Conclusion}

Chiari network can be an incidental finding but can have serious implications on clinical outcomes. Physicians should be aware of the presence of these anomalous structures during clinical decision making. Whenever the diagnosis is not clear, further work up is warranted before making any clinical decision.

\section{References}

[1] Clinical anatomy of the atrial septum with reference to its developmental components. Anderson RH, Webb S, Brown NA. Clin Anat 12:362-37 (1999)

[2] Schneider B, Hofmann T, Justen M. H, Meinertz T. Chiari's network: normal anatomic variant or risk factor for arterial embolic events? Journal of the American College of Cardiology. 1995;26(1):203-210. doi: 10.1016/07351097(95)00144

[3] Bhatnagar KP, Nettleton GS, Campbell FR, Wagner CE, Kuwabara N, Muresian H. Chiari anomalies in the human right atrium. Clin Anat. 2006;19(6):510-6.

[4] Chiari's Network: Normal Anatomic Variant or Risk Factor for Arterial Embolic Events? Brike Schneider MD, Thomas Hoffman MD, Maria H. Justen MD, Thomas Meinertz MD. Handbook of Cerebrovascular Diseases Second Edition, Revised and Expanded (2005)
[5] The Chiari Malformations. R. S. Tubbs, W. J. Oakes (eds), 5 DOI 10.1007/978-1-4614-6369-6_2, Springer Science + Business Media New York 2013

[6] Echocardiographic appearance of the Chiari network: differentiation from right-heart pathology. Werner JA, Cheitlin MD, Gross BW, Speck SM, Ivey TD. Circulation 1981; 63:1104-1109.

[7] Giant Chiari network mimics intracardiac tumor in a case of neurofibromatosis. Koz C, Yokusoglu M, Baysan O, Uzun M. Int J Cardiol 130:488-489 (2008)

[8] Chiari's network: normal anatomic variant or risk factor for arterial embolic events. Schneider B, Hofmann T, Justen MH, Meinertz T. J Am Coll Cardiol 26:203-210(1995)

[9] Chiari network entrapment of thromboembolic: congenital inferior vena cava fil-ter. Goedde TA, Conetta D, Rumisek JD. Ann Thorac Surg 49:317-318(1990)

[10] Catheter entrapment in a Chiari network involving an atrial septal defect. Goldschlager A, Goldschlager N, Brewster H, Kaplan J. Chest 62:345-346(1972)

[11] Prajapat L, Ariyarajah V, Spodick DH. Abnormal atrial depolarization associated with Chiari network? Cardiology. 2007;108(3):214-6.

[12] Schwimmer-okike N, Niebuhr J, Schramek GG, Frantz S, Kielstein H. The Presence of a Large Chiari Network in a Patient with Atrial Fibrillation and Stroke. Case Rep Cardiol. 2016;2016:4839315.

[13] Pothineni K. P, Nanda N. C, Burri M. V, Singh A, Panwar S. $\mathrm{R}$, Gandhari S. Live/real time three-dimensional transthoracic echocardiographic visualization of Chiari network. Echocardiography. 2007;24(9):995-997.

[14] Altbach M. I, Squire S. W, Kudithipudi V, Castellano L, Sorrell V. L. Cardiac MRI is complementary to echocardiography in the assessment of cardiac masses. Echocardiography. 2007;24(3):286-300. 